\title{
NWB-Datenbank Steuerrecht
}

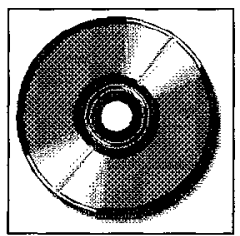

\section{Peter Bilsdorfer}

Der NWB-Verlag ist bei Steuerfachleuten einer der "Wortführer". Das Betätigungsfeld des Verlages liegt bislang im wesentlichen im Bereich der Druckmedien. Marktführend von der Auflage her dürfte hier das seit Jahren eingeführte Loseblattwerk "Neue WirtschaftsBriefe" sein, das dem Verlag auch seinen Namen gab. Dort finden sich mit einer GesamtBuch-Breite von $66 \mathrm{~cm}$ in inzwischen 10 Bänden mit rund 10.000 Seiten, aufgeteilt in unterschiedliche Fachgebiete (nicht nur Steuerrecht, sondern auch Wirtschaftsrecht und Gesetzestexte). Das Gesamtwerk wird ständig über wöchentliche Nachlieferungen ergänzt, deren wesentlicher Teil die sog. Eilnachrichten mit aktuellen Kurzinformationen über neue Rechtsprechung und Verwaltungsanweisungen sind.

\section{Inhalt}

Der NWB-Verlag bietet mit seiner CD-ROM (Stand: Mai 1995) nicht nur den Stoff seines Loseblattwerkes einschließlich der $a b$ 1992 ausgeschiedenen Beiträge und der Eilnachrichten ab 1989 an, sondern ergänzt diesen Be- richtstoff noch um weitere Rechtsquellen, wie

- die Verwaltungsanweisungen in den jew eiligen Richtlinien

- sämtliche in den Verwaltungsanweisungen zitierten $\mathrm{BFH}$-Entscheidungen im Volltext

- sämtliche zitierten BMFSchreiben im Volltext.

Insoweit gleich eine Anregung: Der Verlag sollte überlegen, den Umfang der Verwaltungsanweisungen noch auszudehnen um sämtliche Anweisungen, auf die das Hauptwerk Bezug nimmt. Ein Beispiel: Ein Aufsatz betreffend die Anweisungen für das Straf- und Bußgeldverfahren (Steuer) in Fach 13, S. $793 \mathrm{ff}$., läßt sich noch besser verwerten, wenn der Nutzer den Zugriff auf eben jene Anweisungen hat, die ja nicht die Form eines BMF-Schreibens erhalten haben.

\section{Installation und Nutzeranleitung}

Wie gesagt, die NWB-Datenbank Steuerrecht ist in zwei Versionen erhältlich. Der Rezensent hat ausschließlich die Windows-Version genutzt. Hier war von vornherein die gut gegliederte und an-
Abb. 1: Das Verzeichnis

Peter Bilsdorfer ist Richter am Finanzgericht in Saarbrücken.

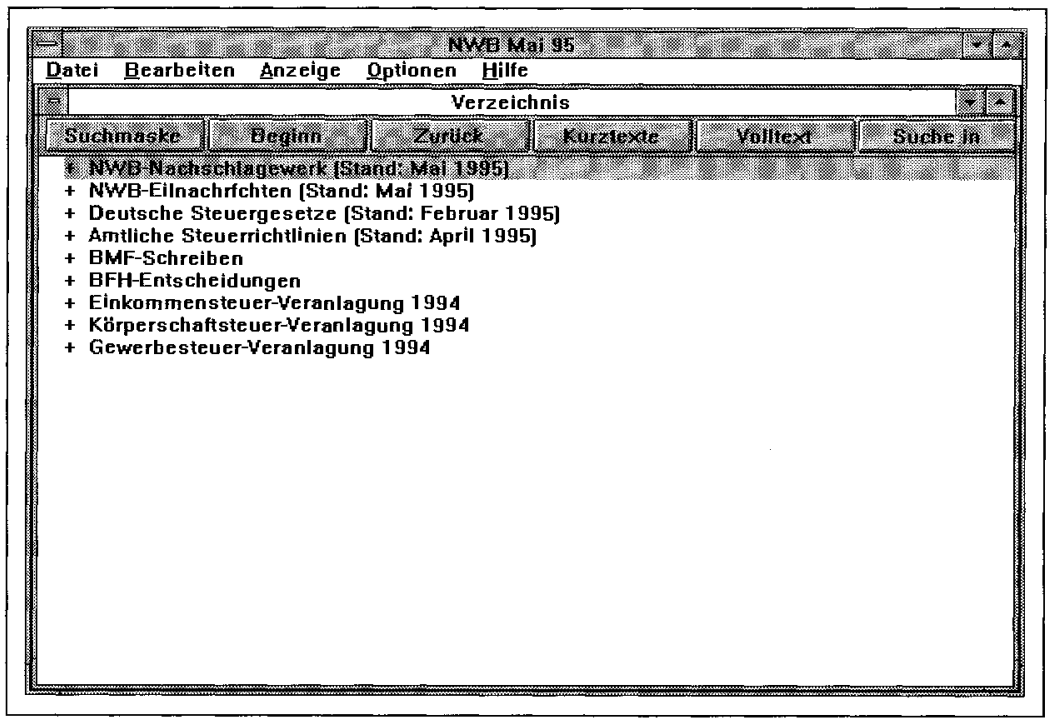

schaulich mit Bildschirmabbildungen versehene, umfassende (und doch nicht überfrachtete) Nutzungsanleitung von Vorteil.

Sie beschreibt vorab die Systemanforderungen (mindestens IBMkompatiblen PC-386; DOS ab Version 3.3.; Windows ab Version 3.1.; $4 \mathrm{MB}$ Arbeitsspeicher; ca. 4 MB Festplattenspeicher, vollständig (einschließlich MSCDEX-Treiber) installiertes CDROM Laufwerk (ISO 9660/High Sierra). Die Installation läßt sich ohne Problem ausführen.

\section{Bedienung}

Die Nutzungsanleitung enthält hier auf knappem Raum bereits das Wesentliche. Für WindowsErfahrene ist die Handhabung von vornherein kein Problem.

Beim ersten Aufruf der Datenbank steht dem Nutzer ein Verzeichnisfenster zur Verfügung (vgl. Abb. 1), das - entsprechend dem Inhaltsverzeichnis eines $\mathrm{Bu}$ ches - einen Überblick des Inhalts der Datenbank verschafft. Man kann alsdann zwei Suchstrategien verfolgen, nämlich

- die Verzeichnisrecherche:

Hier enthält das Verzeichnisfenster das Inhaltsverzeichnis der Datenbank, das im Grunde genommen wie das Inhaltsverzeichnis eines Buches benutzt wird (vgl. Abb. 2 auf der nächsten Seite). Man wählt per Maus oder Pfeiltaste den gewünschten Eintrag und "schlägt" dann die weiterführenden Texte (etwa Amtliche Steuerrichtlinien) auf. Man blättert sich quasi vom Allgemeinen zum Speziellen. Darüber hinaus können einzelne Verzeichnisäste in die Suchmaske übernommen werden, so daß die sodann eingegebenen Begriffe nur noch in diesem Teilbestand der Datenbank gesucht werden.

jur-pc $9+10 / 95$ 
- die Suchmaskenrecherche: Hier erhält der Nutzer eine Suchmaske (vgl. Abb. 3) mit diversen Suchfeldern (Dok.-
Nr., Titel, Verfasser, Dok.Art, Datum, Az/Geschz, Fundstelle, Gesetz/\$S/RL, Text, Zitierung, CDIS-Suche).
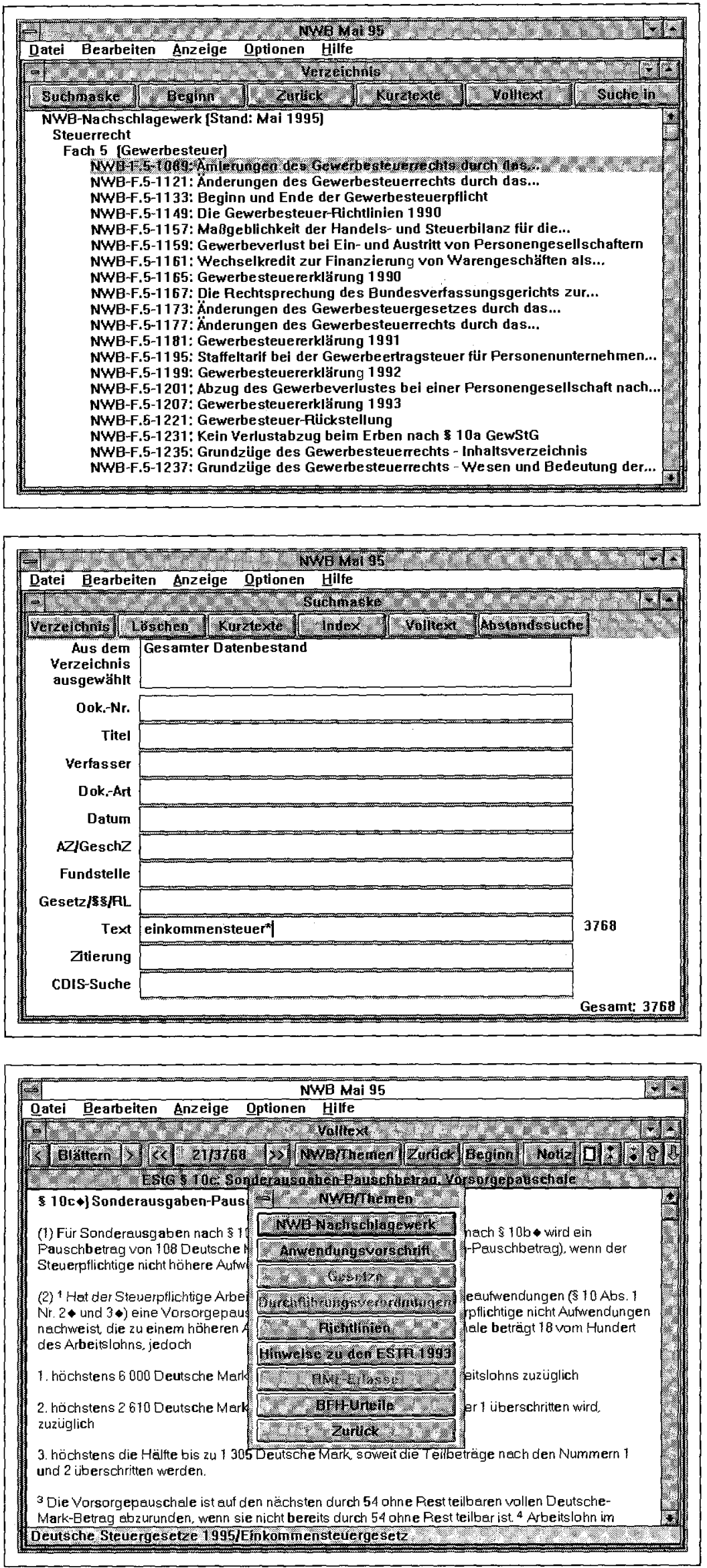

Er kann alsdann ein Suchwort oder aber mehrere eingeben (und ggf. kombinieren) und erhält alsdann die Meldung über die 'Trefferquote. Die gefundenen Dokumente lassen für den raschen Überblick in Form einer Ergebnisliste mit Kurztexten und selbstverständlich auch im Volltext anzeigen.

Auch insoweit ist die Handhabung vollkommen unproblematisch. Soweit in einem aufgerufenen Dokument ein weiterführender Hinweis auf ein weiteres Dokument (etwa eine BFH-Entscheidung) enthalten ist, kann dieses mit einem einfachen Mausklick in Volltextversion einbezogen werden (Abb. 4).

Wichtig auch: Die Exportmöglichkeit. Über das Datei-Menü gelangt man zu den Menüpunkten Drucken und Export und erhält hier die Möglichkeit, markierte Dokumente (etwa einen Auszug aus einem BFH-Urteil) in andere Windowsanwendungen (etwa einen Schriftsatz) zu integrieren.

Insbesondere für steuerliche Berater, aber auch für Firmen (und auch Finanzrichter) von Nutzen: Der Einkommensteuer-Rechner, der bei Eingabe des $\mathrm{zu}$ versteuernden Einkommens sofort die Steuer anhand von Grund- und Splittingtabelle bekanntgibt.

\section{Fazit}

Insgesamt bietet die NWB-Datenbank Steuerrecht sehr viel Erfreuliches. Sie ist benutzerfreundlich gestaltet und auch für den Anfänger ohne weiteres erschließbar. Sie wird jedoch das "gedruckte Wort" nicht entbehrlich machen. So werden treue NWB-Leser schwerlich auf die wöchentlichen Eilnachrichten verzichten wollen. Auf jeden Fall sollten sie aber überlegen, ob sie nicht zur Ergänzung die Datenbank mit ihrem doch erweiterten Angebot heranziehen wollen.
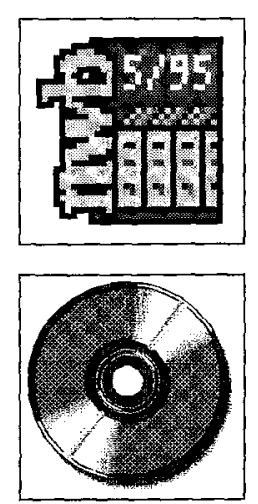

Abb. 2 .

Blättern im Verzeichnis

Abb.3:

Die Suchmaske

Abb. 4:

Hypertexte und Themenquerverweise 